\title{
THE HIGHER COMMUTATOR SUBGROUPS OF A GROUP
}

\section{REINHOLD BAER}

It is not the object of this address to introduce you to new theories or to tell of great discoveries. Quite on the contrary; I intend to speak of unsolved problems and of conjectures. In order to describe these, certain concepts will have to be discussed; and for obtaining a proper perspective it will be necessary to mention a number of theorems, some of them new. The proofs of the latter will be relegated to appendices so that the hurried reader may skip them easily. The bibliography is in no sense supposed to be complete. We just selected convenient references for facts mentioned and beyond that just enough to be a basis for further reading.

1. The hierarchy of invariant subgroups. The subgroups of a group may be classified according to the operations which leave them invariant. There are first the normal subgroups of the group $G$, characterized by the fact that they are transformed into themselves by the inner automorphisms of $G$; and for this reason they had at one time appropriated the term "invariant subgroup." There are next the characteristic subgroups of $G$ which are left invariant by every automorphism of $G$. Clearly not every subgroup of $G$ is normal, unless $G$ belongs to a comparatively special class of groups, the so-called abelian and hamiltonian groups; and neither is in general every normal subgroup characteristic, though this may happen too (for example, in cyclic groups and in simple groups).

These two classes of subgroups are well known, but for our purposes they are too big. There is next the class of subgroups which we shall term for lack of a better name strictly characteristic. A subgroup $S$ of $G$ belongs to this class if $S^{f} \leqq S$ whenever $f$ is an endomorphism $^{1}$ of $G$ and $G^{f}=G$. The distinction between characteristic and strictly characteristic subgroups does not cut very deep, since there exists a very big class of groups with the following property:

(Q) If $f$ is an endomorphism of $G$ such that $G^{f}=G$, then $f$ is an automorphism of $G$.

This postulate $(Q)$ is satisfied by every finite group and more generally by every group satisfying the ascending chain condition for normal subgroups. But the $Q$-groups are not exhausted by the groups

An address delivered before the Annual meeting of the Society in Chicago on November 27, 1943; received by the editors January 5, 1944.

1 An endomorphism of the group $G$ is a single-valued and multiplicative $G$ to $G$ function. 
just mentioned, witness the free groups on a finite number of generators. ${ }^{2}$ However, there exist groups that are not $Q$-groups, for instance the rational numbers modulo 1 and the free groups on an infinity of generators; and an example of a characteristic subgroup which is not strictly characteristic will be constructed in Appendix I.

Finally we have the fully invariant subgroups. ${ }^{3}$ A subgroup $S$ of the group $G$ is termed fully invariant if $S^{\prime} \leqq S$ for every endomorphism $f$ of $G$. Examples of groups whose centers are not fully invariant are easily constructed. 4 On the other hand it is readily verified that the center is always a strictly characteristic subgroup. For if $z$ is an element in the center of the group $G$, and if $G^{f}=G$ is satisfied by the endomorphism $f$ of $G$, then there exists to every element $x$ in $G$ an element $y$ in $G$ satisfying $x=y^{f}$; and we find

$$
z^{f} x=z^{f} y^{f}=(z y)^{f}=(y z)^{f}=y^{f} z^{f}=x z^{f}
$$

so that $z^{f}$ is in the center too. Examples of fully invariant subgroups are obtained by forming the subgroup $G^{n}$ generated by all the $n$th powers of elements in $G$, or the commutator subgroup $(G, G)$ of $G$ which is generated by all the commutators $(x, y)=x^{-1} y^{-1} x y$. More generally it may be said that the "word subgroups" in the sense of B. Neumann and P. Halls are fully invariant whereas the "marginal subgroups" may only be shown to be characteristic.

The importance of characteristic subgroups stems from the fact that they themselves and their quotient groups are group invariants. But if we compare different groups, we have to have a method for deciding which are "corresponding" invariants of the different groups. ${ }^{6}$ For it would not do to compare the structure of $G^{3}$ with the structure of $H^{5}$ or the order of $G$ with the maximum order of the elements in $H$. We indicate one such possibility of comparing the fully invariant subgroups of different groups. If $S$ is a subgroup of the group $G$, then we denote by $(G \rightarrow H ; S)$ the subgroup of the group $H$ which is generated by all the elements $s^{f}$ for $s$ an element in $S$ and $f$ a homomorphism of $G$ into $H$. It is readily seen that $(G \rightarrow H ; S)$ VIII].

2 For proofs of this theorem see F. Levi $[1$, p. 95] and W. Magnus $[1$, p. 276,

Criteria for Q-groups may be found in R. Baer [2], D. I. Fouxe-Rabinowitsch [1], and A. Malcew [1]. Numbers in brackets refer to the Bibliography at the end of the paper.

3 This concept is due to F. Levi [1].

4 See, for example, Appendix I below.

${ }^{5} \mathrm{P}$. Hall [2], B. Neumann [1].

- See in this context the functors discussed by Eilenberg and MacLane [2]. 
is always a fully invariant subgroup of $H$; and if $S$ is a fully invariant subgroup of $G$, then we have

$$
(\mathrm{H} \rightarrow \mathrm{G} ;(\mathrm{G} \rightarrow \mathrm{H} ; \mathrm{S})) \leqq \mathrm{S} .
$$

Equality we cannot always expect. But if $G$ and $H$ are both free groups on an infinity of generators, then equality holds, so that the fully characteristic subgroups of $G$ and $H$ form isomorphic partially ordered sets. ${ }^{7}$

\section{APPENDIX I: EXISTENCE OF CHARACTERISTIC, BUT NOT STRICTLY CHARACTERISTIC SUBGROUPS}

Let $F$ be a finite group which is not abelian. Then $F$ contains a cyclic subgroup $T$ which is not part of the center of $F$. Denote by $Z$ a cyclic group whose order equals the order of $T$ and denote by $G(0)$ the direct product of $F$ and $Z$. It is clear that $Z$ is part of the center of $G(0)$ and that there exists an endomorphism $f(0)$ of $G(0)$ which maps $F$ upon 1 and which effects an isomorphism of $Z$ upon $T$.

Since $G(0)$ is a finite group, there exists a free group $G(1)$ on a finite number of generators and a homomorphism $f(1)$ of $G(1)$ upon the (full) group $G(0)$. Denote by $G(i)$ for $1<i$ groups isomorphic to $G(1)$ and by $f(i)$ an isomorphism of $G(i)$ upon $G(i-1)$ for $1<i$. Finally let $G$ be the free product ${ }^{8}$ of all the groups $G(i)$ for $0 \leqq i$. Then there exists $^{9}$ one and only one endomorphism $f$ of $G$ which induces $f(i)$ in $G(i)$ for $0 \leqq i$; and it is clear that $G=G^{f}$.

Suppose now that $B$ is a finite subgroup of $G$. Then it follows from a theorem on the subgroups of free products ${ }^{10}$ that $B$ is the free product of a free group $V$ and of groups of the form ${ }^{11} B \cap x^{-1} G(i) x$. But $B$ is a finite group and therefore it cannot contain a free group different from 1 , nor can it be the free product of two groups different from 1 . On the other hand free groups do not possess finite subgroups different from 1 , since subgroups of free groups are free. ${ }^{12}$ Thus we have shown the following fact.

If $B$ is a finite subgroup of $G$, then $B \leqq x^{-1} G(0) x$ or $x B x^{-1} \leqq G(0)$ for suitable $x$ in $G$.

Denote now by $C$ the subgroup of $G$ which is generated by the

${ }^{7}$ Baer [3].

${ }^{8}$ For the theory of free products see, for example, Baer and Levi [1].

${ }^{9}$ If we had formed the direct product of the groups $G(i)$ instead of forming their free product, then it would have been impossible to construct this endomorphism $f$.

${ }^{10}$ Baer and Levi [1, p. 392].

"11 By $S \cap T$ we designate the crosscut of the sets $S$ and $T$.

${ }^{12}$ By Schreier's theorem. 
center $D$ of $G(0)$ and by all its conjugates in $G$. If $g$ is an automorphism of $G$, then $G(0)^{\circ}$ is a finite subgroup of $G$. Hence it follows from the lemma just proved that $G(0)^{o}=y^{-1} G(0) y$ for some $y$ in $G$. Consequently $D=y D^{o} y^{-1}$, since $D$ is a characteristic subgroup of $G(0)$, and since the succession of $g$ and of the inner automorphism $s \rightarrow y s y^{-1}$ of $G$ induces an automorphism of $G(0)$. But from this fact one readily deduces that $C$ is a characteristic subgroup of $G$.

From the theorems ${ }^{13}$ on subgroups of free products one deduces now that $C$ is the free product of $D$ and of some conjugates to $D$ in $G$. Thus $D$ is a free factor of $C$ and is consequently the crosscut of $C$ and $G(0)$. This shows in particular that $T$ is not part of $C$. But $T$ is part of $C^{f}$, since $D^{f}=D^{f(0)}=T$. Hence

\section{$C$ is not a strictly characteristic subgroup of $G$.}

2. Commutator subgroups. If $S$ and $T$ are subsets of the group $G$, then we denote by $(S, T)$ the subgroup generated by all the commutators $(s, t)=s^{-1} t^{-1} s t$ for $s$ in $S$ and $t$ in $T$. If $S$ and $T$ are fully invariant subgroups of $G$, then so is $(S, T)$. Thus it is possible to use this operator for the inductive definition of fully invariant subgroups. We mention two important instances.

The derived series: $G^{(o)}=G, G^{(i+1)}=\left(G^{(i)}, G^{(i)}\right)$.

The lower central series: ${ }^{\circ} G=G,{ }^{i+1} G=\left(G,{ }^{i} G\right)$.

These are not the only possibilities. For instance, one could define a series $G(i)$ of fully invariant subgroups by the following recursion formulas:

$$
G(0)=G, G(1)=(G, G), G(i+1)=(G(i), G(i-1)) \text { for } 0<i .
$$

But so far only the derived and the lower central series seem to have proved interesting. A systematic method of constructing higher commutator subgroups has been evolved by P. Hall. ${ }^{14}$ But his constructions are too involved to be sketched in a few words.

The calculus of commutator subgroups is based on the following simple formulas, which are easily verified by direct computation..$^{15}$

$$
\begin{aligned}
(x, y) & =(y, x)^{-1} . \\
(x y, z) & =y^{-1}(x, z) y(y, z)=(x, z)((x, z), y)(y, z) . \\
(x, y z) & =(x, z) z^{-1}(x, y) z=(x, z)(z,(y, x))(x, y) .
\end{aligned}
$$

If $X, Y, Z$ are subgroups of the group $G$, then one deduces from these

\footnotetext{
${ }^{18}$ Baer and Levi [1, p. 396, Folgerung 3].

${ }^{14}$ Hall [1], in particular p. 43-48.

${ }^{15}$ Hall $[1$, p. 43].
} 
formulas the following results.

$$
\begin{aligned}
(X, Y) & =(Y, X) . \\
(X,(Y, Z)) & \leqq(Y,(Z, X))(Z,(X, Y)) .
\end{aligned}
$$

An induction argument using the definitions of derived and lower central series as well as formulas (4) and (5) leads now to the inequalities

$$
\left({ }^{i} G,{ }^{j} G\right) \leqq{ }^{i+j+1} G, G^{(i)} \leqq{ }^{2^{i}-1} G .
$$

Thus the derived series decreases much more rapidly than the lower central series. Another significant distinction between these two series is the fact that $G^{(i+1)}$ is a fully invariant subgroup of $G^{(i)}$ whereas ${ }^{i+1} G$ need not be a fully invariant subgroup of ${ }^{i} G$. A property common to both these series is the fact that the quotient groups of successive members are abelian.

Special properties of such series of fully invariant subgroups are invariant properties of the underlying groups and may therefore be used for characterizing special classes of groups. We give two examples of such properties.

(S) 1 is the crosscut of the groups $G^{(i)}$.

(N) 1 is the crosscut of the groups ${ }^{i} G$.

It is an immediate consequence of formula (6) that every $N$-group is an $S$-group, though the converse is not true. In fact, finite $S$-groups are just the soluble groups whereas finite $N$-groups are exactly the nilpotent groups. ${ }^{18}$ Finite nilpotent groups are known to be direct products of $p$-groups; and this theorem may be generalized as follows.

Every $N$-group without elements of order ${ }^{19} 0$ is the direct product of (finite or infinite) p-groups. ${ }^{20}$

In Appendix II we prove this theorem and we construct an example of a $p$-group $P$ satisfying $P^{(2)}=1$, though it is not an $N$-group, showing that the converse of the above theorem does not hold.

$N$-groups generated by a finite number of elements may be shown to be $Q$-groups (as defined in $\$ 1$ ), ${ }^{21}$ though there exist $S$-groups generated by two elements which are not $Q$-groups. ${ }^{22}$

${ }^{16}$ Hall $[1, \mathrm{p} .47$, Theorem 2.3].

${ }^{17}$ Hall $[1$, p. 53, Theorem 2.51 and p. 54, Theorem 2.54].

${ }^{18}$ See, for example, Baer [1], where further references may be found.

${ }^{19}$ An element in a group is said to be of order 0 if it generates an infinite cyclic group.

${ }^{20}$ A p-group is a group all of whose elements are of order a power of $p$.

${ }^{21}$ See, for example, Fouxe-Rabinowitsch [1].

${ }^{22}$ For proofs of these facts, cp. Baer [2]. 
Finally we indicate a method ${ }^{23}$ for proving that a given group $G$ is an $N$-group. Suppose that the ring $R$ contains an identity element 1 (satisfying $r 1=1 r=r$ for every $r$ in $R$ ), that 0 is the crosscut of the powers $P^{i}$ of the two-sided ideal $P$ in $R$, and that $G$ is a multiplicative group of elements in $R$ meeting the requirement:

$g \equiv 1$ modulo $P$ for $g$ in $G$.

If $g$ is an element in $G$, then there exist uniquely determined elements $g^{\prime}, g^{\prime \prime}$ in $P$ such that $g=1+g^{\prime}$ and $g^{-1}=1+g^{\prime \prime}$; and $g^{\prime \prime}$ belongs to $P^{i}$ if $g^{\prime}$ is an element in $P^{i}$. If $g, h$ are elements in $G$, and if $h^{\prime}, h^{\prime \prime}$ belong to $P^{i}$, then

$$
\begin{aligned}
(g, h) & =\left(1+g^{\prime \prime}\right)\left(1+h^{\prime \prime}\right)\left(1+g^{\prime}\right)\left(1+h^{\prime}\right) \\
& \equiv 1+g^{\prime \prime}+h^{\prime \prime}+g^{\prime}+h^{\prime}+g^{\prime \prime} g^{\prime}+h^{\prime \prime} h^{\prime} \text { modulo } P^{i+1} \\
& \equiv 1 \text { modulo } P^{i+1} .
\end{aligned}
$$

Thus one is able to prove by complete induction the following fact: If $g$ belongs to ${ }^{i} G$, then $g \equiv 1$ modulo $P^{i+1}$. Consequently $G$ itself is an $N$-group, since the crosscut of the ideals $P^{i}$ is 0 .

If $T$ and $S \leqq T$ are normal subgroups of the group $G$, then we denote by $S \div T$ the set of all the elements $x$ in $G$ satisfying $(x, T) \leqq S$. It is readily seen ${ }^{24}$ that the set $S \div T$ is a normal subgroup of $G$; and if $S$ and $T$ are characteristic subgroups of $G$, so is their commutator quotient $S \div T$. The best known example is the center $1 \div G$ of the group $G$ or more generally:

The upper central series: $Z_{0}(G)=1, Z_{i}(G)=Z_{i-1}(G) \div G$ for $0<i$.

Interesting relations between upper and lower central series of a finite $p$-group have been discovered by P. Hall. ${ }^{25}$ There are, however, fundamental differences, since the one series is descending, the other ascending, the one consists of fully invariant subgroups, the other of strictly characteristic subgroups which need not be fully characteristic.

In analogy to the definition of $N$ - and $S$-groups one is led to the $Z$-groups, meeting the following requirement. ${ }^{26}$

(Z) Every element in $G$ is contained in at least one $Z_{i}(G)$.

A finite group is known to be a $Z$-group if, and only if, it is an $N$-group. But in general no such relation holds. The non-abelian free groups are examples of $N$-groups with centers equal to 1 , and there

${ }^{23}$ This method has been introduced by W. Magnus [1] who used it to prove that free groups are $N$-groups.

${ }_{24}^{4}$ Baer [3], Zassenhaus [1].

${ }^{25}$ Hall $[1$, p. 53, Theorem 2.51].

${ }^{26}$ Baer [1]. 
exist $p$-groups with this property. ${ }^{27}$ An example of a $Z$-group, not an $N$-group, will be constructed in Appendix II. ${ }^{28}$

\section{APPENDIX II : NILPOTENCY OF $N$-GROUPS}

If $x$ and $y$ are elements of order a power of $p$ in the $N$-group $G$ without elements of order 0 , then the order of $x y$ is of the form $i p^{j}$ where $i$ is an integer prime to $p$. We denote by $W$ the subgroup generated by $x$ and $y$; and we put $(x y)^{p^{j}}=z$ so that $z$ is an element of the order $i$ prime to $p$. From ${ }^{k} W \leqq{ }^{k} G$ we infer that 1 is the crosscut of the subgroups ${ }^{k} W$.

We are going to prove by complete induction with regard to $k$ that $W /{ }^{k} W$ is a finite group for every $k$. This is certainly true for $k=0$; and thus we assume its validity for $k-1$. Since $W$ is generated by a finite number of elements, and since $W /^{k-1} W$ is finite, we deduce from the Reidemeister-Schreier method ${ }^{29}$ that ${ }^{k-1} W$ is generated by a finite number of elements. Consequently ${ }^{k-1} W /{ }^{k} W$ is an abelian group, generated by a finite number of elements whose orders are different from 0 . This implies the finiteness of ${ }^{k-1} W /{ }^{k} W$, proving that $W /{ }^{k} W$ is finite too.

Since $W /{ }^{k} W$ is a finite group whose lower central series ends with 1 , and since $W /{ }^{k} W$ is generated by two elements of order a power of $p$, it follows from well known theorems ${ }^{30}$ that $W /{ }^{k} W$ is a $p$-group. Hence $z \equiv 1$ modulo ${ }^{k} W$ for every positive $k$. Thus $z$ belongs to the crosscut of the subgroups ${ }^{k} W$. But this crosscut is 1 , showing that $z=1$. Therefore we have proved the following fact.

If $x$ and $y$ are elements of order a power of $p$ in the $N$-group $G$ without elements of order 0 , then $x y$ is an element of order a power of $p$.

If we denote by $G(p)$ the set of all the elements of order a power of $p$ in $G$, then it follows from the fact just established that $G(p)$ is a subgroup of $G$. Hence $G(p)$ is a fully invariant and therefore a normal subgroup of $G$. If $p$ and $q$ are different primes, then $G(p)$ and $G(q)$ are normal subgroups of $G$ which have only the identity in common. Consequently $r s=s r$ for $r$ in $G(p)$ and $s$ in $G(q)$. Now it is readily seen that $G$ is the direct product of its primary components $G(p)$, as we desired to prove.

The condition that the group $G$ be an $N$-group, though indispensa-

${ }^{27}$ Baer [1, pp. 412, 413, Example 3.4].

${ }^{28}$ For another example of a $Z$-group, not an $N$-group, see Baer [1, p. 406 , Example 2.5].

${ }^{29} \mathrm{See}$, for example, Baer $[1$, p. $396(1.3)]$ or Zassenhaus [1, p. 108].

${ }^{30}$ See, for example, Zassenhaus $[1$, p. 107]. 
ble for the preceding proof, is not a necessary condition, as will be seen from the following example of a p-group which is not an $N$-group.

Denote by $B$ the abelian group generated by elements $b, b(1)$, $b(2), \cdots, b(i), \cdots$, subject to the relations:

$$
1=b^{p}, b=b(i)^{p i} \text { for every positive } i \text {. }
$$

It is readily seen that the crosscut $B^{p^{\omega}}$ of all the groups $B^{p^{i}}$ is the cyclic group of order $p$, generated by $b$, and that $B / B^{p^{\omega}}$ is the direct product of a cyclic group of order $p$ by a cyclic group of order $p^{2} \ldots$ by a cyclic group of order $p^{i} \ldots$ and that the elements $b(i)$ represent a basis of $B / B^{p^{\omega}}$.

Denote by $g(i)$ the automorphism of $B$ which maps $b(i)$ upon $b(i)^{1+p}$ (and thus leaves $b$ invariant) and which leaves all the $b(j)$ for $j \neq i$ invariant. It is obvious that the automorphism $g(i)$ is of order $p^{i}$ and that the automorphisms $g(i)$, for positive $i$, generate an abelian group.

Let finally $G$ be the group obtained by adjoining to $B$ elements $t(i)$ subject to the relations:

$$
1=t(i)^{p i}, t(i)^{-1} x t(i)=x^{g(i)} \text { for } x \text { in } B \text { and positive } i \text {. }
$$

This group $G$ is an extension of the group $B$ by the group of automorphisms, generated by the $g(i)$ 's.

Since $G / B$ and $B$ are both abelian p-groups, $G$ itself is a $p$-group. Furthermore $G^{(2)}=1$. One verifies inductively that ${ }^{i} G=B^{p^{i}}$ and that the crosscut of the subgroups ${ }^{i} G$ is therefore $B^{p^{\omega}} \neq 1$. Thus $G$ has been shown to be a $p$-group, an $S$-group, but not an $N$-group.

Furthermore one verifies by complete induction that $Z_{i}(G)$, the $i$ th term in the upper central series of $G$, is generated by the elements $t(j)$ for $0<j<i$ and by the elements $x$ in $B$ which satisfy $x^{p^{i}}=1$. Thus $G$ is a $Z$-group.

3. Burnside's problem. Before the impact of topology on group theory made itself felt, group theory was concerned almost exclusively with the study of finite groups. Practically the first fact noticed by the student of finite groups is that the orders of the elements in a finite group are bounded and different from 0 . This latter property defines a larger class of groups, which we are going to discuss now.

If the orders of the elements in the group $G$ are bounded, then there exists the 1.c.m. of the orders of all the elements in $G$. If $m$ is this number, then $x^{m}=1$ for every $x$ in $G$. Every group $G$ is the homomorphic image of a free group $F$. If $N$ is the kernel ${ }^{31}$ of the homomor-

s1 The kernel of the homomorphism $f$ consists of those elements which are mapped upon 1 by $f$. 
phism mapping $F$ upon $G$, then $x^{m}=1$ for $x$ in $G$ is equivalent to ${ }^{32}$ $F^{m} \leqq N$; and thus $G$ is not only the homomorphic image of the free group $F$, but even of $F / F^{m}$. These free groups reduced modulo $m$ may therefore be considered as universal groups for the class of groups under consideration. There is little known about their nature ${ }^{38}$ and Burnside's problem might be stated somewhat loosely as the problem of determining the structure of $F / F^{m}$.

Let us start with a simple observation. The commutator $(x, y)$ may be seen readily to equal

$$
(x, y)=x^{-1} y^{-1} x y=\left(x^{-1} y^{-1} x\right)^{2} x^{-2}(x y)^{2}
$$

so that we have in every group $G$ the following relation:

$$
(G, G) \leqq G^{2} \text {. }
$$

This shows that $F / F^{2}$ is abelian and this makes it possible to answer completely any question we might have concerning the structure of these groups $F / F^{2}$.

It may easily be derived from a not at all trivial result of $F$. Levi and B. L. van der Waerden ${ }^{84}$ that

$$
{ }^{3} G \leqq G^{3} ;
$$

and thus one is led to the question whether there exists to every positive integer $n$ an integer $n^{\prime}$ such that

$$
{ }^{\prime} G \leqq G^{n} .
$$

The answer to this question is clearly in the negative, since we have noted before that ${ }^{\prime \prime} G \leqq G^{n}$ implies that $G / G^{n}$ is the direct product of its $p$-components whereas not every group satisfying $G^{n}=1$, for some $n$, is the direct product of its $p$-components. As a matter of fact a still stronger result may be proved.

If the free group $F$ is not abelian, and if $F / F^{n}$ is an $N$-group, then $n$ is a power of a prime.

For a proof of this theorem see Appendix III. For prime powers $n$ we prove in Appendix III a still stronger result; to wit:

If there exists an integer $n^{\prime}$ such that ${ }^{\prime \prime} G \leqq G^{n}$ for every group $G$, then $n$ is a prime.

\footnotetext{
${ }^{32} F^{m}$ is the subgroup generated by all the $m$ th powers of elements in the group $F$.

${ }^{83}$ This is the reason why so much more emphasis is laid upon the commutator subgroups than upon these subgroups $F^{m}$.

84 Levi and van der Waerden $[1$, p. 155].
} 
Whether or not the converse of either of these theorems holds true is not known at present; and a decision would constitute an important step towards a solution of Burnside's problem.

The problem as Burnside himself conceived it was stated in a more limited fashion than we have considered it, since he imposed the additional restriction upon the groups under consideration that they should be generated by a finite number of elements. For these groups he has stated two famous conjectures..$^{35}$

(I) $F / F^{n}$ is finite for every free group $F$ of finite rank..$^{36}$

(II) $F / F^{n}$ is soluble for $n$ odd and $F$ a free group of finite rank.

These conjectures are certainly true for $n=2$ and 3 .

The conjecture (II) may be restated in a somewhat more precise form, since $\left(F / F^{n}\right)^{(i)}=\left(F^{n} F^{(i)}\right) / F^{n}$.

(II') If $n$ is an odd integer and if $m$ is a positive integer, then there exists an integer $k=k(n, m)$ such that

$$
F^{(k)} \leqq F^{n} \text { for } F \text { a free group of rank } m \text {. }
$$

A similar restatement of (I) is impossible because of the existence of simple groups. But for prime powers $n$ this is possible, if one recalls that the lower central series of a finite $p$-group ends with the identity and that ${ }^{i}\left(F / F^{n}\right)=\left({ }^{i} F F^{n}\right) / F^{n}$; and thus we obtain the following conjecture.

( $\left.\mathrm{I}^{\prime}\right)$ If $n$ is a power of a prime, and if $m$ is a positive integer, then there exists an integer $h=h(n, m)$ such that

$$
{ }^{h} F \leqq F^{n} \text { for } F \text { a free group of rank } m \text {. }
$$

That $\left(\mathrm{I}^{\prime}\right)$ and $\left(\mathrm{II}^{\prime}\right)$ imply the finiteness of $F / F^{n}$ is a consequence of the following easily verified fact. If the group $G$ is generated by a finite number of elements, and if every element in $G$ is of finite order, then $G /{ }^{i} G$ and $G / G^{(i)}$ are both finite groups. But $\left(\mathrm{I}^{\prime}\right)$ and $\left(\mathrm{II}^{\prime}\right)$ for prime powers are essentially equivalent conjectures, as may be seen from the following theorem, a proof of which may be found in Appendix III.

If $G$ is a p-group, generated by a finite number of elements, then there exist to every positive integer $i$ integers $i^{\prime}, i^{\prime \prime}$ such that

$$
i^{\prime} G \leqq G^{(i)}, \quad G^{\left(i^{\prime \prime}\right)} \leqq{ }^{i} G .
$$

But in case these conjectures should not be verified, then this theo-

${ }^{35}$ Burnside [1].

${ }^{26}$ The rank $r(F)$ of the free group $F$ is the number of elements in any free set of generators of $F$. It is at the same time the rank of the free abelian group $F /(F, F)$. 
rem still asserts that $a$-group which is generated by a finite number of elements is an $N$-group if, and only if, it is an S-group.

The importance of these statements may be seen from the following almost obvious equivalence.

If $n$ is a power of a prime, and if $F$ is a free group of finite rank, then the following two conditions are necessary and sufficient for finiteness of $F / F^{n}$.

(a) $F / F^{n}$ is an $N$-group.

(b) The lower central series of $F / F^{n}$ is finite.

But by the preceding remarks we may substitute for (a) and (b) the following conditions.

(a') $F / F^{n}$ is an $S$-group.

$\left(b^{\prime}\right)$ The derived series of $F / F^{n}$ is finite.

If $n$ is a prime power such that $F / F^{n}$ is an $N$ - (or $S$-)group for every free group $F$ of finite rank, then one may verify the corresponding statement for every $F / F^{n}$ with free $F$. Furthermore it is immediately seen that $F / F^{n}$ is an $N$-group if, and only if, $F^{n}$ is the crosscut of the subgroups ${ }^{i} F F^{n}$ for all the integers $i$. The fact that free groups are $N$-groups may be considered an encouraging sign. But more is known. For Zassenhaus [2] proved a theorem from which it is easy to derive the fact that 1 is the crosscut of the groups ${ }^{i} F F^{p^{i}}$ for $p$ a prime and $i=1,2, \ldots$. All this, however, does not suffice to prove (a). Thus it may be a consolation to know that it is possible to substitute for (a) and $\left(a^{\prime}\right)$ the following weaker conditions without impairing the validity of the above statements.

$\left(\mathrm{a}^{*}\right)$ If $\left(F / F^{n}\right) /{ }^{i}\left(F / F^{n}\right) \neq 1$, then ${ }^{i+1}\left(F / F^{n}\right)<i\left(F / F^{n}\right)$.

$\left(\mathrm{a}^{\prime *}\right)$ If $\left(F / F^{n}\right) /\left(F / F^{n}\right)^{(i)} \neq 1$, then $\left(F / F^{n}\right)^{(i+1)}<\left(F / F^{n}\right)^{(i)}$.

Let us turn now to explaining the significance of condition (b) and its equivalent $\left(\mathrm{b}^{\prime}\right)$. Denote by $N$ the crosscut of all the subgroups $i\left(F / F^{n}\right)$. For $n$ a power of a prime, $N$ is at the same time the crosscut of the subgroups $\left(F / F^{n}\right)^{(i)}$. The following fact is now readily verified.

If condition (b) is satisfied by the prime power $n$, if the free group $F$ is of finite rank, then the following conditions are necessary and suffcient for the group $G$ to be a homomorphic image of the group $\left(F / F^{n}\right) / N$ :

(i) $G$ is finite.

(ii) $G^{n}=1$.

(iii) $G$ may be generated by $r(F)$ elements.

Condition (b) has been verified for $n=5$ and $r(F)=2$ by P. Hall..$^{87}$

${ }^{37}$ For a proof see Grün [2]. 
Both conditions $\left(a^{*}\right)$ and (b) have been investigated recently with rather powerful tools. ${ }^{38}$ So far, however, the results obtained have not been of that striking nature that lends itself to easy narration.

\section{APPENDIX III : THE LOWER CENTRAL SERIES OF FREE GROUPS MODULO $n$}

THEOREM 1. If $F$ is a non-abelian free group and $n$ a positive integer such that $F / F^{n}$ is an $N$-group, then $n$ is a power of a prime.

PROof. Suppose that $n=n^{\prime} n^{\prime \prime}$ is the product of the two relatively prime integers $n^{\prime}$ and $n^{\prime \prime}$ both of which are different from 1 . Denote by $S$ the direct product of $n^{\prime}$ cyclic groups each of which is of order $n^{\prime \prime}$, and let $s(1), \cdots, s\left(n^{\prime}\right)$ be a basis of $S$. There exists one and only one automorphism $k$ of $S$ which maps $s(i)$ for $0<i<n^{\prime}$ upon $s(i+1)$ and $s\left(n^{\prime}\right)$ upon $s(1)$. This automorphism $k$ is clearly of order $n^{\prime}$. Consequently there exists a group $T$ which is obtained by adjoining to $S$ an element $t$ subject to the relations:

$$
t^{n^{\prime}}=1, t^{-1} s t=s^{k} \text { for } s \text { in } S .
$$

This group $T$ is an extension of the abelian group $S$ of order $n^{\prime \prime n^{\prime}}$ by the cyclic group $T / S$ of order $n^{\prime}$. If $x$ is an element in $T$, then $x^{n^{\prime}}$ is in $S$ and $x^{n}=\left(x^{n^{\prime}}\right)^{n^{\prime \prime}}=1$, since $S^{n^{\prime \prime}}=1$. Furthermore it is readily seen that $T$ may be generated by the two elements $t$ and $s(1)$. But $T$ is not the direct product of its primary components, since the two elements $t$ and $s(1)$ of the relatively prime orders $n^{\prime}$ and $n^{\prime \prime}$ do not permute.

Since $F$ is a non-abelian free group, its rank is at least 2 and consequently there exists a homomorphism $h$ of $F$ upon $T$. If $H$ is the kernel of the homomorphism $h$, then we deduce $F^{n} \leqq H$ from $T^{n}=1$; and thus $h$ induces a homomorphism of $F / F^{n}$ upon $T$. Since $T$ is not the direct product of its primary components, neither is $F / F^{n}$, as homomorphisms map primary components upon primary components. We proved in Appendix II that $N$-groups without elements of order 0 are the direct products of their primary components. Hence $F / F^{n}$ is not an $N$-group if $n$ is not a power of a prime.

THEOREM 2. If there exists an integer $n^{\prime}$ such that ${ }^{\prime} G \leqq G^{n}$ for every group $G$, then $n$ is a prime.

Proof. Suppose that $n, n^{\prime}$ are positive integers such that ${ }^{n^{\prime}} F \leqq F^{n}$ for every free group $F$ of finite rank (this hypothesis which is weaker than the one enunciated in the theorem will be shown to be suffi-

${ }^{88}$ Grün [1], Magnus [1, 2], Witt [1], Zassenhaus [2]. 
cient). Then $F / F^{n}$ satisfies ${ }^{n}\left(F / F^{n}\right)=1$. Hence $F / F^{n}$ is an $N$-group and it follows from Theorem 1 that $n$ is a power of a prime.

It has been shown elsewhere ${ }^{39}$ that there exists a group $G$ with the following properties:

(i) Both $G /(G, G)$ and $(G, G)$ are direct products of cyclic groups of order $p$.

(ii) The center of $G$ is equal to 1 .

From (i) one infers in particular that $G^{(2)}=1$ and that $G^{p^{2}}=1$. Thus there exists a representation of $G$ as a quotient group of a free group: $G=F / N$ satisfying $F^{2} \leqq N$.

Suppose now that the prime power $n=p^{m}$ is not a prime, so that $1<m$. Then it follows from a preceding remark that $F^{n} \leqq N$ and that $G$ is a homomorphic map of $F / F^{n}$. Suppose now that the element $x$ in $F$ belongs to ${ }^{\prime} F$. Then there exists a free factor $V$ of $F$ which is a free group of finite rank such that $x$ belongs to ${ }^{\prime \prime} V$. Now we may apply the hypothesis stated at the beginning of the proof, namely that $n^{\prime} V \leqq V^{n}$. Thus $x$ belongs to $V^{n}$ and therefore to $F^{n}$, and we have shown: ${ }^{\prime} F \leqq F^{n} \leqq N$. But this shows that the lower central series of $G=F / N$ is finite and ends with 1 , a fact that is readily seen to contradict (ii). This contradiction proves that $n$ is a prime.

Let us term the positive integer $n$ Burnsidean if $F / F^{n}$ is finite for every free group $F$ of finite rank. Then we may prove the following converse of Theorem 1.

THEOREM $1^{\prime}$. If $n$ is a Burnsidean prime power, and if $F$ is a free group, then $F / F^{n}$ is an $N$-group.

Proof. If $x$ is an element not 1 in $F$, then there exists a homomorphism $h$ of $F$ upon a free group $V$ of finite rank such that $x^{h} \neq 1$, since $F$ is the free product of two free groups $V$ and $W$, the first one of which contains $x$ and is of finite rank. This homomorphism $h$ maps $F^{i}$ upon $V^{i}$ and ${ }^{i} F$ upon ${ }^{i} V$ for every positive $i$. If $x$ were contained in the crosscut ${ }^{\omega} F$ of the subgroups ${ }^{i} F$, then $x^{h}$ would belong to the crosscut ${ }^{\omega} V$ of the subgroups ${ }^{i} V$. But $n$ is a Burnsidean prime power. Thus $V / V^{n}$ is finite and of order a power of a prime. Hence there exists an integer $w$ such that ${ }^{w} V \leqq V^{n}$. Since $x^{h}$ belongs to ${ }^{\omega} V$, it belongs to ${ }^{w} V$ and therefore to $V^{n}$. But this implies that $x$ belongs to $F^{n}$, and hence we have shown ${ }^{\omega} F \leqq F^{n}$, a fact that is equivalent to the assertion: $F / F^{n}$ is an $N$-group.

We note that it would have been sufficient for our proof to make the hypothesis : $F / F^{n}$ is an $N$-group for every free group $F$ of finite rank,

${ }^{39}$ Baer [1, pp. 412, 413, Example 3.4]. 
a hypothesis that is considerably weaker than the assumption that $n$ is Burnsidean.

As an application of Theorem 1' we prove a converse of the theorem proved in Appendix II.

THEOREM 3. Suppose that the positive integer $n$ is the product of Burnsidean prime powers, and that the group $G$ satisfies $G^{n}=1$. Then $G$ is the direct product of its primary components if, and only if, $G$ is the homomorphic image of an $N$-group $H$ satisfying $H^{n}=1$.

Proof. It is a consequence of a theorem established in Appendix II that $N$-groups $H$ satisfying $H^{n}=1$ are the direct products of their primary components. Homomorphisms map direct products of $p$ groups upon direct products of $p$-groups, proving the sufficiency of our condition.

Suppose conversely that $G$ is the direct product of its primary components. If $n=p(1)^{m(1)} \cdots p(k)^{m(k)}$ with $0<m(i), 0<k$, then $G$ is the direct product of groups $G(i)$ satisfying $G(i)^{n(i)}=1$ for $n(i)=p(i)^{m(i)}$. The group $G(i)$ may be represented as a quotient group $F(i) / M(i)$ of a free group $F(i)$; and clearly $F(i)^{n(i)} \leqq M(i)$. Thus $G(i)$ is the homomorphic image of $F(i) / F(i)^{n(i)}$. By hypothesis $n(i)$ is a Burnsidean prime power and thus it follows from Theorem $1^{\prime}$ that $R(i)=$ $F(i) / F(i)^{n(i)}$ is an $N$-group. If $R$ is the direct product of the $N$-groups $R(i)$, then $R^{n}=1, R$ is an $N$-group and $G$ is the homomorphic image of $R$, proving our contention.

THeOREM 4. If $G$ is a p-group, generated by a finite number of elements, then there exist to every positive integer i integers $i^{\prime}, i^{\prime \prime}$ such that

$$
i^{\prime} G \leqq G^{(i)}, \quad G^{\left(i^{\prime \prime}\right)} \leqq{ }^{i} G .
$$

PROOF. It is a consequence of a theorem quoted in $\$ 1$ that we may select as $i^{\prime \prime}$ any integer satisfying $i<2^{i^{\prime \prime}}$.

Since ${ }^{i} G /{ }^{i+1} G$ and $G^{(j)} / G^{(j+1)}$ are abelian groups the orders of whose elements are powers of the prime $p$, since such abelian groups are finite if they are generated by a finite number of elements, one proves inductively by the usual application of the Reidemeister-Schreier method ${ }^{40}$ that $G /{ }^{i} G$ and $G / G^{(i)}$ are finite groups. Thus $G / G^{(i)}$ is a finite $p$-group, and such groups are known to have the property that their lower central series is finite and ends with 1 . Hence there exists an integer $i^{\prime}$ such that $i^{\prime}\left(G / G^{(i)}\right)=1$ and this is equivalent to

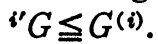

${ }^{10}$ See footnote 29. 
4. Invariants of the Hopf type. The group invariants discussed so far were subgroups and quotient groups of subgroups of the group under discussion. But there exist other possibilities of connecting groups with groups in an invariant fashion. An instance is the automorphism group of a group.

A group may be given either by inner properties or by some sort of representation. An example of the first kind of definition is the following one: the abelian group of order $p^{n}$ the orders of whose elements are 1 and $p$. An example of the second kind is the customary definition of the symmetric group of degree $n$. Though it is certainly more desirable to characterize a group by inner properties, it is more common to define it by some sort of representation, and very often a suitable representation makes a group better accessible to treatment.

H. Hopf ${ }^{41}$ has recently discovered a group invariant derived from a representation of a group as a quotient group. He showed that for normal subgroups $N$ of free groups $F$ the quotient group

$$
(F, F) /(F, N)
$$

depends only on the structure of $F / N$, and is an invariant of $F / N$. Now the groups $F / F^{n}$, discussed in $\S 3$, are defined by their representations only. Thus these Hopfian invariants and their generalizations may be a tool in their investigation.

We indicate a new proof of Hopf's invariance theorem which has the advantage of being a convenient basis for many important generalizations. To simplify notation we restrict ourselves to a discussion of the lower central series, which is quite typical.42

Suppose that $M$ is a normal subgroup of the group $H$. Then $H / M$ is a representation of a certain group $G$. We define inductively subgroups ${ }_{i} M$ by the formulas : ${ }_{0} M=M,{ }_{i} M=\left(H,{ }_{i-1} M\right)$. Then every ${ }_{i} M$ is a normal subgroup of $H$, and we note the following inequalities:

$$
{ }_{i} M \leqq{ }^{i} H,{ }_{i} M \leqq{ }_{i-1} M \text { and } M \leqq{ }_{i} M \div{ }^{i} H .
$$

1. Let us consider an endomorphism $h$ of the group $H$, satisfying $x^{h} \equiv x$ modulo $M$. Of $h$ we say then that it induces the identity in $H / M$. Assume now that we have shown already that $h$ induces the identity in ${ }^{i} \mathrm{H} /{ }_{i} M$. Then a simple application of formulas (2), (3) of $\$ 2$ shows that $h$ induces the identity in ${ }^{i+1} H /{ }_{i+1} M$.

2. Consider now two representations $H / M$ and $K / N$ of the same group $G$ and homomorphisms $h, k$ of $H$ into $K$ and $K$ into $H$ respectively with the following property: $h$ induces an isomorphism $a$ of

${ }^{41}$ Hopf [1].

42 Baer [3]. 
$H / M$ upon $K / N$ and $k$ induces the isomorphism $a^{-1}$ of $K / N$ upon $H / M$.

This hypothesis implies in particular $M^{h} \leqq N$ and $N^{k} \leqq M$; and one verifies readily that ${ }^{i} H^{h} \leqq i k,{ }^{i} K^{k} \leqq i H,{ }_{i} M^{h} \leqq{ }_{i} N,{ }_{i} N^{k} \leqq_{i} M$. But $h k$ induces the identity in $H / M$; and thus it follows from what we have shown under 1 that $h k$ induces the identity in every $i H / i M$. From this fact and the previously stated inequalities one deduces immediately that $h$ and $k$ induce reciprocal isomorphisms between ${ }^{i} H /{ }_{i} M$ and ${ }^{i} \mathrm{~K} /{ }_{i} N$.

This implies in particular that the isomorphism $a$ maps $\left({ }_{i} M \div{ }^{i} H\right) / M$ upon $\left({ }_{i} N \div{ }^{i} K\right) / N$.

3. We say now that two representations $H / M$ and $K / N$ of the group $G$ are similar if every isomorphism between $H / M$ and $K / N$ may be induced by homomorphisms. Using this terminology the result obtained under 2 implies the following generalization of Hopf's invariance theorem: ${ }^{i} H /{ }_{i} M$ and $\left({ }_{i} M \div{ }^{i} H\right) / M$ are invariants of the class of similar representations of $G$ to which $H / M$ belongs.

It should be mentioned that not every representation belongs to a class of similar representations.

That this theorem is really a generalization of Hopf's theorem is a consequence of the fairly obvious fact that all representations of the group $G$ as a quotient group of a free group (or of a free abelian group, ${ }^{43}$ or of a free group reduced modulo $n$, and so on) are similar.

The range of applicability of these invariants seems to be fairly wide. So far they have been used mainly to show that homomorphisms meeting certain requirements are isomorphisms. ${ }^{42}$

As these invariants have not been defined in an invariant fashion, one may desire an invariant definition. This may be obtained either by using a normal form of the representation which is trivial as far as it can be done and not very interesting, or by a properly invariant definition which does not refer to any representation. This latter problem seems to be fairly deep and has been solved so far apparently in only one instance. ${ }^{44}$

\section{BIBLIOGRAPHY}

R. BAER

1. Nilpotent groups and their generalizations, Trans. Amer. Math. Soc. vol. 47 (1940) pp. 393-434.

2. Groups without proper isomorphic quotient groups. To appear in Bull. Amer. Math. Soc.

3. Representations of groups as quotient groups and their invariants. In course of preparation.

${ }^{43}$ Eilenberg and MacLane [1, p. 765, Lemma 4.3].

${ }^{44}$ Eilenberg and MacLane [3]. 
R. BAER AND F. LEVI

1. Freie Produkte und ihre Untergruppen, Compositio Math. vol. 3 (1936) pp. 391-398.

W. BURNSIDE

1. On an unsettled question in the theory of discontinuous groups, Quart. J. Math. Oxford Ser. vol. 33 (1902) pp. 230-238.

S. Eilenberg and S. Maclane

1. Group extensions and homology, Ann. of Math. (2) vol. 43 (1942) pp. 757-831.

2. Natural isomorphisms in group theory, Proc. Nat. Acad. Sci. U. S. A. vol. 28 (1942) pp. 537-543.

3. Relations between homology and homotopy groups, Proc. Nat. Acad. Sci. U. S. A. vol. 29 (1943) pp. 155-158.

D. I. FouXE-RABINOWITSCH

1. On the determinators of an operator of the free group, Rec. Math. (Mat. Sbornik) N. S. vol. 7 (1940) pp. 197-206.

O. GRÜN

1. Über eine Faktorgruppe freier Gruppen. I, Deutsche Mathematik vol. 1 (1936) pp. 772-782.

2. Zusammenhang zwischen Potenzbildung und Kommutatorbildung, J. Reine Angew. Math. vol. 182 (1940) pp. 158-177.

P. Hall

1. A contribution to the theory of groups of prime power order, Proc. London Math. Soc. vol. 36 (1934) pp. 29-95.

2. Verbal and marginal subgroups, J. Reine Angew. Math. vol. 182 (1940) pp. 156158.

H. HopF

1. Fundamentalgruppe und zweite Bettische Gruppe, Comment. Math. Helv. vol. 14 (1942) pp. 257-309.

F. LEVI

1. Über die Untergruppen der freien Gruppen, Math. Zeit. vol. 37 (1933) pp. 90-97.

F. LEVI AND B. L. VAN DER WAERDEN

1. Über eine besondere Klasse von Gruppen, Abh. Math. Sem. Hamburgischen Univ. vol. 9 (1932) pp. 154-158.

W. MAGNUS

1. Beziehungen zwischen Gruppen und Idealen in einem speziellen Ring, Math. Ann. vol. 111 (1935) pp. 259-280.

2. Über Beziehungen zwischen hoheren Kommutatoren, J. Reine Angew. Math. vol. 177 (1937) pp. 105-115.

3. Neuere Ergebnisse ibber auflösbare Gruppen, Jber. Deutschen Math. Verein. vol. 47 (1937) pp. 69-78.

4. Über freie Faktorgruppen und freie Untergruppen gegebener Gruppen, Monatshefte fur Mathematik und Physik vol. 47 (1938) pp. 307-313.

5. Allgemeine Gruppentheorie, Enzyklopaedie der mathematische Wissenschaften I, 4, 9; 2. Aufl., 1939.

A. MALCEW

1. On isomorphic matrix representations of infinite groups, Rec. Math. (Mat. Sbornik) N. S. vol. 8 (1940) pp. 421-422 (405-421).

B. H. NeUManN

1. Identical relations in groups. I, Math. Ann. vol. 114 (1937) pp. 506-552. 
K. REIDEMEISTER

1. Einfilhrung in die kombinatorische Topologie, Braunschweig, 1932.

E. WirT

1. Treue Darstellung Liescher Ringe, J. Reine Angew. Math. vol. 177 (1937) pp. 152-160.

H. Zassenhaus

1. Lehrbuch der Gruppentheorie, I, Hamburger Mathematische Einzelschriften vol. 21 (1937).

2. Ein Verfahren, jeder endlichen p-Gruppe einen Lie-ring mit der Charakteristik $p$ zuzuordnen, Abh. Math. Sem. Hamburgischen Univ. vol. 13 (1940) pp. 200-207.

UNIVERSITY OF ILLINOIS 